J. Dairy Sci. 95:3837-3851

http://dx.doi.org/10.3168/jds.2011-5241

(C) American Dairy Science Association ${ }^{\circledR}, 2012$.

\title{
Candidate mechanisms underlying atypical progesterone profiles as deduced from parameter perturbations in a mathematical model of the bovine estrous cycle
}

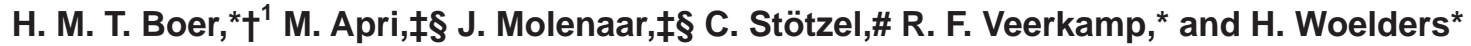 \\ *Animal Breeding and Genomics Centre, Wageningen UR Livestock Research, 8200 AB Lelystad, the Netherlands \\ †Adaptation Physiology Group, Department of Animal Sciences, Wageningen University, 6700 AH Wageningen, the Netherlands \\ ¥Biometris, Department of Mathematical and Statistical Methods, Wageningen University, 6700 AC Wageningen, the Netherlands \\ §Netherlands Consortium for Systems Biology, 1090 GE Amsterdam, the Netherlands \\ \#Computational Systems Biology Group, Department of Numerical Analysis and Modeling, Konrad-Zuse-Zentrum für Informationstechnik Berlin, \\ Takustraße 714195 Berlin, Germany
}

\begin{abstract}
The complex interplay of physiological factors that underlies fertility in dairy cows was investigated using a mechanistic mathematical model of the dynamics of the bovine estrous cycle. The model simulates the processes of follicle and corpus luteum development and its relations with key hormones that interact to control these processes. Several factors may perturb the regular oscillatory behavior of a normal estrous cycle, and such perturbations are likely the effect of simultaneous changes in multiple parameters. The objective of this paper was to investigate how multiple parameter perturbation changes the behavior of the estrous cycle model, so as to identify biological mechanisms that could play a role in the development of cystic ovaries. Cystic ovaries are a common reason for reproductive failure in dairy cows, but much about the causes of this disorder remains unknown. We investigated in which region of the parameter space the model predicts a normal cycle, and when a progesterone pattern occurred with delayed ovulation (indicating a cystic follicle) or delayed luteolysis (indicating a persistent corpus luteum). Perturbation of the initial values for all parameters simultaneously showed 2 specific parameter configurations leading to delayed ovulation or delayed luteolysis immediately. The most important parameter changes in these 2 configurations involve the regulation of corpus luteum functioning, luteolytic signals, and GnRH synthesis, suggesting that these mechanisms are likely involved in the development of cystic ovaries. In the multidimensional parameter space, areas exist in
\end{abstract}

Received December 7, 2011.

Accepted March 8, 2012.

${ }^{1}$ Corresponding author: Marike.Boer@wur.nl which the parameter configurations resulted in normal cycles. These areas may be separated by areas in which irregular cycle patterns occurred. These irregular patterns thus mark the transition from one stable (normal) situation to another. Interestingly, within a series, there were some cycles with delayed ovulation and some with delayed luteolysis in these patterns. This could represent a situation of resumption of normal cyclicity (e.g., after parturition). In conclusion, the method of parameter perturbation used in the present study is an effective tool to find parameter configurations that lead to progesterone profiles associated with delayed ovulation and delayed luteolysis. Thereby, the model helps to generate hypotheses regarding the underlying cause of the development of cystic ovaries, which could be investigated in future experiments.

Key words: mathematical model, estrous cycle, progesterone, cystic ovaries

\section{INTRODUCTION}

Recently, we developed a deterministic mathematical model that describes the dynamics of the bovine estrous cycle as a set of linked differential equations. The model generates cyclical fluctuations of hormones, follicles, and corpora lutea in estrous cycles of approximately $21 \mathrm{~d}$ for cows with a normal estrous cycle. The parameters in the model represent kinetic properties of the system with regard to synthesis, release, and clearance of hormones, and growth and regression of follicles and corpora lutea (Boer et al., 2011b). Model simulations are in line with empirical observations, indicating that the model is a credible representation of reproductive physiology.

Several factors may disturb the regular oscillatory nature of the bovine estrous cycle. In the model, this translates to the question how much the parameter values could be perturbed without a change in the 
qualitative behavior of the model. This is interesting to assess, as it could identify biological mechanisms that are critical in the regulation of the bovine estrous cycle. The effects of changing specific parameters one at a time on the behavior of the model of the bovine estrous cycle was analyzed in Boer et al. (2011a), but in the reality of biological systems it is more likely that a perturbation is the effect of simultaneous changes in multiple physiological parameters, as can be simulated in the model by multi-parameter perturbations.

Several existing methods for multi-parameter robustness analysis (i.e., multi-dimensional sensitivity analysis; reviewed in Ghaemi et al., 2009) suffer from computational limitations because of the exponential increase of the number of combinations in systematic variation of multiple parameters. Here, we use an approach that is especially designed to be efficient for systems with a large number of parameters (Apri et al., 2010). More specifically, we wanted to identify parameters that are involved in the formation of cystic ovaries, an important cause of reproductive failure in dairy cows (Garverick, 1997; Braw-Tal et al., 2009). Cystic follicles or persistent corpora lutea develop when the dominant follicle or the corpus luteum (CL) fails to regress and maintains steroidogenesis. Metabolic and endocrine adaptations to high milk yield and negative energy balance are known to increase the risk of the formation of cystic ovaries, but the exact pathogenesis is unclear (reviewed in Vanholder et al., 2006). Identification of parameter perturbations involved in the formation of cystic ovaries could help in understanding which biological mechanisms play a role. Likely, there is not one exact pathogenesis, but different configurations of unfavorable physiological settings, resulting in cystic follicles or persistent corpora lutea. The modeling approach used here allows identification of different candidate scenarios.

The objective of this paper was to assess how physiological parameters may disturb the regular oscillatory nature of the bovine estrous cycle, and to identify mechanisms that could play a role in the formation of cystic ovaries. To obtain this, the progesterone $(\mathbf{P} 4)$ profiles simulated by the model were evaluated on the occurrence of delayed ovulation, indicating a cystic follicle, or delayed luteolysis, indicating a persistent CL.

\section{MATERIALS AND METHODS}

Our approach to search for mechanisms that could play a role in the formation of cystic ovaries can be summarized as follows. Using a mechanistic mathematical model of the bovine estrous cycle, we investigated in which region in the parameter space a normal cycle is simulated and when a P4 pattern associated with delayed luteolysis or delayed ovulation occurred. We defined 4 criteria for $\mathrm{P} 4$ profiles, as generated by the model, to categorize estrous cycles as normal or abnormal. By perturbing the initial values for all 60 parameters simultaneously, the parameter space was then scanned to detect where a normal cycle is predicted and where a pattern of delayed ovulation or delayed luteolysis occurs. By doing so, we obtained an estimation of the robustness region of the model (i.e., the whole region of parameter values for which a normal cycle is predicted). The model, the method of parameter perturbation, and the criteria for a $\mathrm{P} 4$ profile of a normal estrous cycle are described in more detail in the next paragraphs.

\section{Brief Description of the Model}

We used the deterministic mathematical model of the bovine estrous cycle originally described by Boer et al. (2011a) and Boer et al. (2011b) with the modifications introduced more recently by Stötzel et al. (2012). The model describes the dynamics of the bovine estrous cycle as a set of linked differential equations, including the process of follicle and CL development and the relations with key hormones that interact to control these processes (Figure 1). In the model, follicles are not modeled separately, and Foll represents the capacity of follicles present at any moment to produce estradiol (E2) and inhibin, rather than follicular size. Likewise, CL represents the capacity of the CL to produce P4. The model generates estrous cycles of approximately 21 d, with 2 or 3 peaks (depending on the parameterization) of FSH and 2 or 3 corresponding waves of follicle growth and, hence, E2 and inhibin production. Progesterone, which is high during the first wave(s) of the cycle, decreases as the CL regresses under influence of PGF2 $\alpha$ release from the uterus. Prostaglandin $F_{2 \alpha}$ is stimulated by oxytocin $(\mathbf{O T})$ and OT receptor $(\mathbf{O T R}$, representing the overall OT-mediated mechanism in the endometrium involved in the production of $\mathrm{PGF}_{2 \alpha}$ ). The effect of $\mathrm{PGF}_{2 \alpha}$ on the CL is mediated by several local factors, such as endothelin-1-system, cytokines, and nitric oxide, included in the model as interovarian factors. When the CL has regressed, the last follicular wave results in increasing E2 levels. This causes a surge of GnRH and hence LH, which triggers ovulation. The part of Foll regressing due to the LH surge (i.e., the ovulated follicle) is preserved in the system and forms the developing CL in the next estrous cycle. The model contains 15 differential equations (Figure 1) with 60 parameters. The initial parameterization (default settings) results in estrous cycles with 3 waves of follicle 


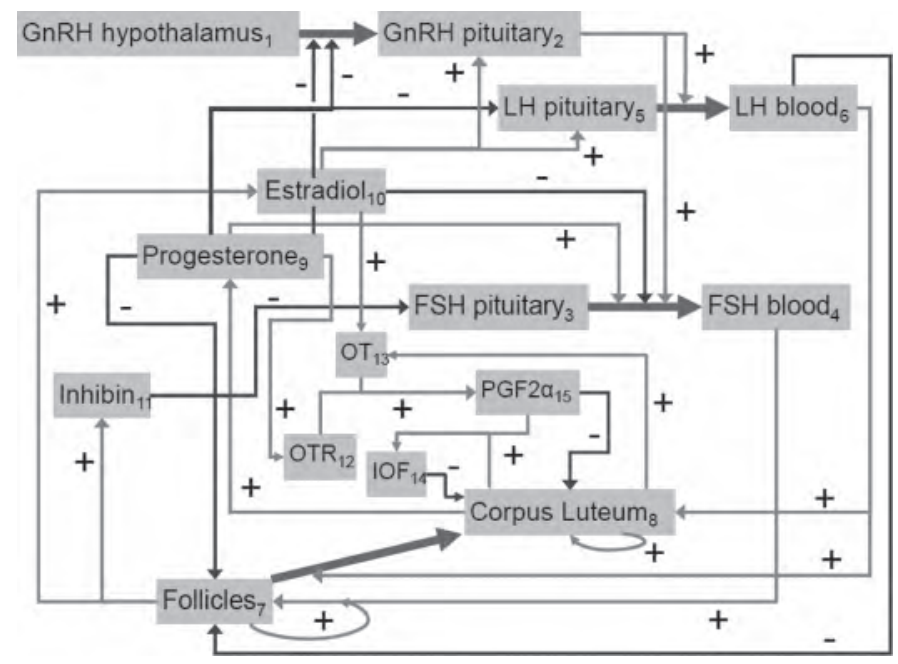

Figure 1. Mechanisms of the model of the bovine estrous cycle, in which the boxes represent the 15 components for which a differential equation was derived. The numbers in the subscripts indicate the equations (Table $\mathrm{A} 1$ of the Appendix) that belong to each component. At the arrows (indicating specific parameterizations of a given equation), stimulating and inhibiting effects are denoted by + and - , respectively. $\mathrm{OT}=$ oxytocin; $\mathrm{OTR}=\mathrm{OT}$ receptor; $\mathrm{IOF}=$ interovarian factors.

growth (Figure 2). A complete overview of all equations and initial parameter values can be found in the Appendix.

\section{Estimation of the Robustness Region}

The initial values (i.e., default settings or baseline values) of the 60 model parameters (the so-called nominal set, given in Table A3 of the Appendix) result in a normal periodic solution with 3 waves of follicle development per cycle (Figure 2). However, it is expected that in a certain region in the 60-dimensional space around this nominal set the system still shows the oscillatory behavior that is characteristic for a normal cycle. We refer to this region as the robustness region. The model parameters differ in sensitivity, and this oscillatory behavior is more easily perturbed by some parameter combinations than by others. We assume that this represents that certain parts of the biological system are more sensitive to environmental or genetic changes than others. Using criteria for a normal estrous cycle (as defined in the next section), the method described in Apri et al. (2010) was adjusted and applied to find an approximation of the robustness region. The present method is highly efficient for systems with a high-dimensional parameter space, as the number of simulations scales linearly and not exponentially with the number of parameters.

The adjusted method can be explained as follows. First, a gradient vector, $\nabla g$, of which the entries represent the sensitivity of luteal phase length, $g$ (as defined in the next section), to changes in the parameter val-
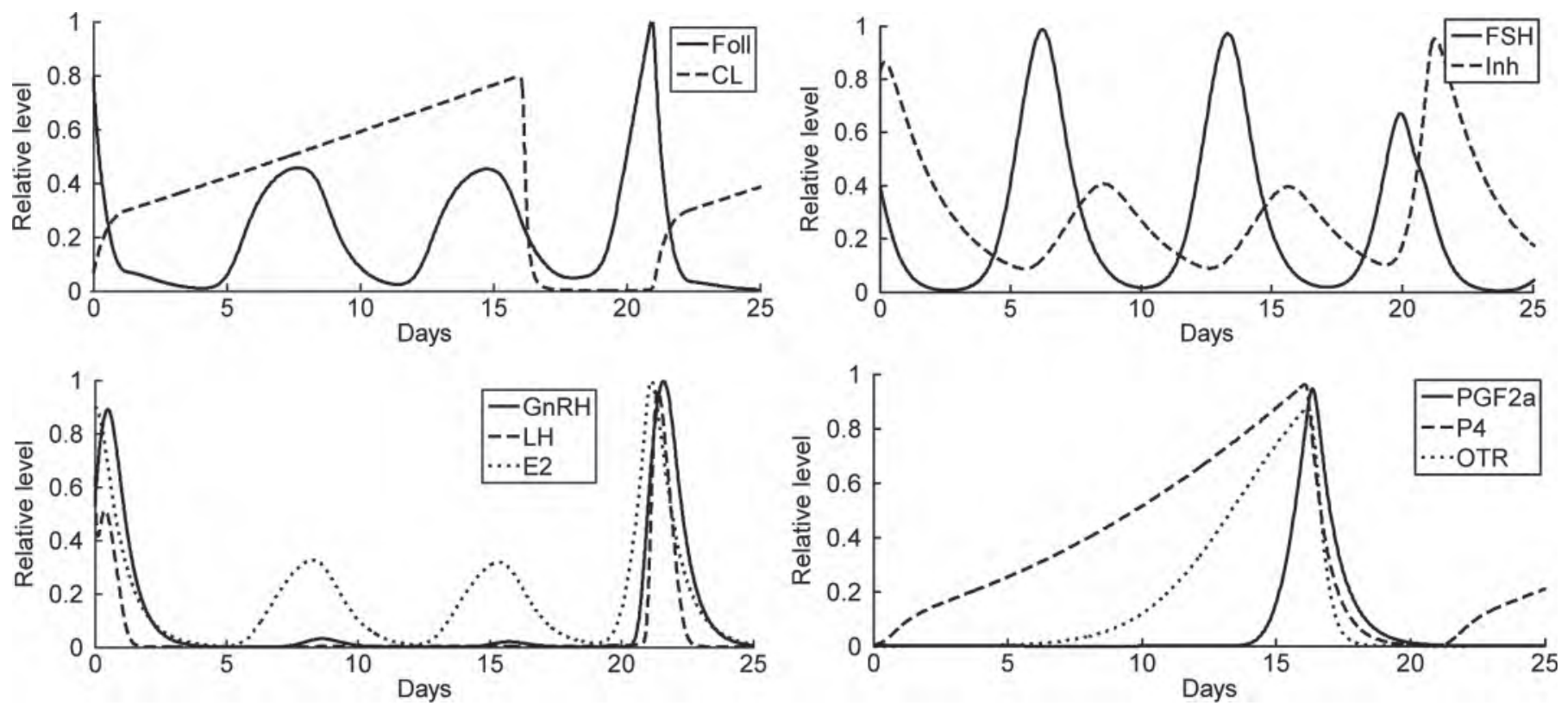

Figure 2. The initial model parameterization (default settings) results in estrous cycles with 3 waves of follicular growth. The equations are expressed on a relative scale to simplify parameter estimation, and therefore, the y-axis of the figures is dimensionless. Foll = follicular function; $\mathrm{CL}=$ corpus luteum function; Inh $=$ inhibin; E2 = estradiol; P4 = progesterone; OTR $=$ oxytocin receptor. 
ues, $k$, was calculated according to equation 24 in Apri et al. (2010):

$$
\nabla g=\left(\frac{\delta g}{\delta k_{1}}, \ldots, \frac{\delta g}{\delta k_{m}}\right)
$$

This gradient vector is calculated numerically using

$$
\frac{\delta g}{\delta k_{j}} \approx \frac{g\left(k_{1}, \ldots, k_{j}+\varepsilon, \ldots, k_{m}\right)-g\left(k_{1}, \ldots, k_{m}\right)}{\varepsilon}, j=1, \ldots, m,
$$

in which $\varepsilon$ (a small positive number) is made smaller and smaller until convergence is reached. Here, the function $g\left(k_{1}, \ldots, k_{m}\right)$ represents the luteal phase length as a function of the parameter set $\mathbf{k}=\left(k_{1}, \ldots\right.$ ,$\left.k_{m}\right)$. This gradient vector was normalized by defining the sensitivity vectors:

$$
S_{j}=\frac{\delta g}{\delta k_{j}} \frac{k_{j}}{g}, j=1, \ldots, m
$$

The advantage of this normalization is that it yields a dimensionless sensitivity vector $\mathbf{S}=\left(S_{1}, S_{2}, \ldots, S_{m}\right)$. This sensitivity vector represents the direction in which the luteal phase length changes most. Next, other perturbation directions were constructed such that they are not only perpendicular to the first direction $\mathbf{S}$, but also perpendicular to each other. These other perturbation directions are calculated by the Gram-Schmidt method. The whole procedure leads to, in total, 60 vectors, which are all mutually perpendicular and include the sensitivity vector $\mathbf{S}$. However, to get a first impression of the robustness region, initially a 2-dimensional robustness analysis was carried out for the 2 parameters to which the luteal phase length was most sensitive. Then, starting from the nominal set $\left(\mathbf{k}_{0}\right)$, all parameter values were perturbed in the directions of these perpendicular vectors to detect where along these directions the model behavior shows a qualitative transition. For each vector, one may move in 2 opposite directions, so in fact one may deviate from the nominal set $\left(\mathbf{k}_{0}\right)$ in 120 directions. Note that if one moves in one of those directions, the values of all 60 parameters may change simultaneously, as these directions are not parallel to the parameter axes. The perturbation along a certain direction was stopped if one of the parameters approached zero (because all parameters should be positive), if $\mathrm{P} 4$ levels became too high (as defined in the next section), or if the $\mathrm{P} 4$ profiles met the definition for delayed ovulation or delayed luteolysis (see also next section). The model was simulated for 10 successive luteal phases.

\section{Definition of Progesterone Profiles for a Normal Cycle}

A normal estrous cycle consists of a period of elevated P4 levels (luteal phase) of about $15 \mathrm{~d}$ and a period of low P4 levels (inter-luteal interval or estrous phase) of about $6 \mathrm{~d}$ (Darwash et al., 1998). A cystic follicle or persistent CL occurs when the follicle or CL, respectively, fails to regress and persists on the ovary. Consequently, alterations in hormone patterns occur. Delayed ovulation involves the formation of a cystic follicle, leading to a prolonged interluteal interval (i.e., an extended period of low P4 serum levels). Delayed luteolysis involves the formation of a persistent CL, leading to a prolonged luteal phase length (i.e., an extended period of high P4 serum levels). Both abnormalities are thus represented by atypical $\mathrm{P} 4$ patterns. Abnormal P4 profiles were classified by Darwash et al. (1998), and the classification has subsequently been applied by others (Garmo et al., 2009; Petersson et al., 2006; Vanholder, 2005). We used these classifications to define when delayed ovulation and delayed luteolysis occur in the model when we perturb the model parameter values (Figure 3). The relative threshold levels used in the model can be linked to the levels reported by Darwash et al. (1998). In brief, the 4 criteria for a normal estrous cycle are 1) values $>0$ for all parameters, 2) maximum relative $\mathrm{P} 4$ levels below 2.00, 3) luteal phase length of 9 to $19 \mathrm{~d}$, and 4) interluteal interval of maximum $12 \mathrm{~d}$. Delayed ovulation in the model is defined as P4 concentration below the relative level 0.15 (where the peak luteal P4 level in the model is initially about 1.00) for more than $12 \mathrm{~d}$. Delayed luteolysis in the model is defined as P4 concentration above the relative level 0.15 for more than $19 \mathrm{~d}$. Simulations with the model (not presented) show that an estrous cycle has only 2 waves when P4 levels are above 0.15 for $9 \mathrm{~d}$ (resulting in a cycle length of $17 \mathrm{~d}$ ), and this was taken as minimum duration of the luteal phase. Upper boundary for P4 levels was set at 2.00, which represents the maximum $\mathrm{P} 4$ levels reported by Darwash et al. (1998). Consequently, a cycle is considered normal when $\mathrm{P} 4$ levels are between 0.15 and 2.00 for 9 to $19 \mathrm{~d}$ in the luteal phase and below 0.15 for less than $12 \mathrm{~d}$ in the interluteal interval. With these definitions, the initial length of the interluteal interval and the luteal phase length in the model are similar to the mean lengths in Darwash et al. (1998). Whether the model predicts a normal cycle or not was 


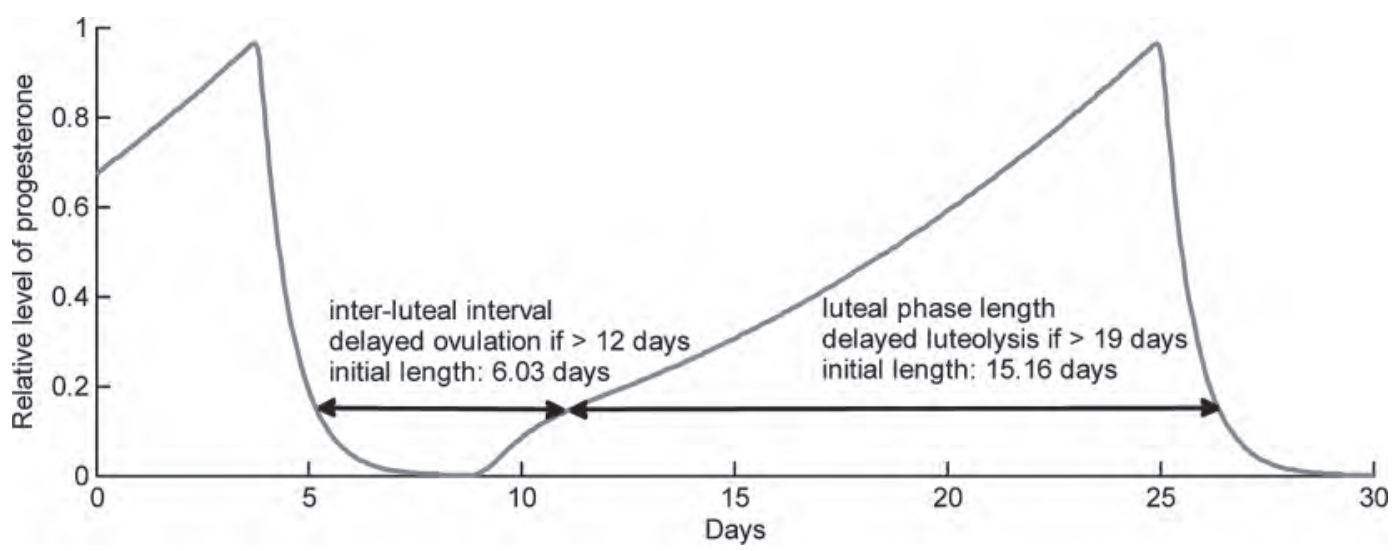

Figure 3. Illustration of the luteal phase parameters analyzed in the model. The figure shows the progesterone (P4) levels at the initial parameterization (nominal set).

calculated based on P4 levels only, but we also evaluated the model solution for E2 and LH to see if these plots were in line with the decision made by the model based on $\mathrm{P} 4$. Thus, a quantitative validation of model performance was done with $\mathrm{P} 4$ levels against Darwash et al. (1998), and a visual assessment of E2 and LH was done as intuitive confirmation of the results.

\section{RESULTS}

\section{Robustness Region for a Normal Estrous Cycle}

The sensitivity analysis showed that the most sensitive parameter with respect to luteal phase length was parameter 41 (maximum increase of CL stimulated by itself), followed by parameter 51 (maximum increase of OTR stimulated by $\mathrm{P} 4$ ), as a change in these parameters induced the largest change in luteal phase length. To get a first impression of the robustness region, a 2-dimensional robustness analysis was carried out in the plane spanned by these 2 most sensitive parameters in the system. The cross-section of the robustness region within this plane is presented in Figure 4. This cross-section, colored in gray, was obtained by scanning the parameter space $\left(k_{41}, k_{51}\right)$, while fixing the other parameters at their nominal value. For all points in the gray region the model predicts a normal cycle. Obviously, the nominal parameter set $\mathbf{k}_{0}$ lies inside the robustness region. From Figure 4 it can be observed that the robustness region of the model might be quite complicated. Its cross-section with the $\left(k_{41}, k_{51}\right)$ plane shows 2 distinct branches where a normal estrous cycle is predicted, and between the branches the model predicts a pattern of delayed ovulation or delayed luteolysis. The nominal parameter set $\mathbf{k}_{0}$ is situated in the lower branch, close to the boundary of the robustness region. Because here only 2 parameters are allowed to vary at the same time, it is relatively easy to capture the exact shape of this part of the robustness region. However, if all parameters are allowed to vary simultaneously, a more effective way to estimate the robustness region is required.

The analysis was continued by estimating the 60 -dimensional robustness region applying the method described in the Methods section. All perturbations were started from the nominal set $\left(\mathbf{k}_{0}\right)$. For convenience, all perturbation direction vectors were scaled such that each of them has 1 unit length. To give an impression of these vectors, the components of perturbation

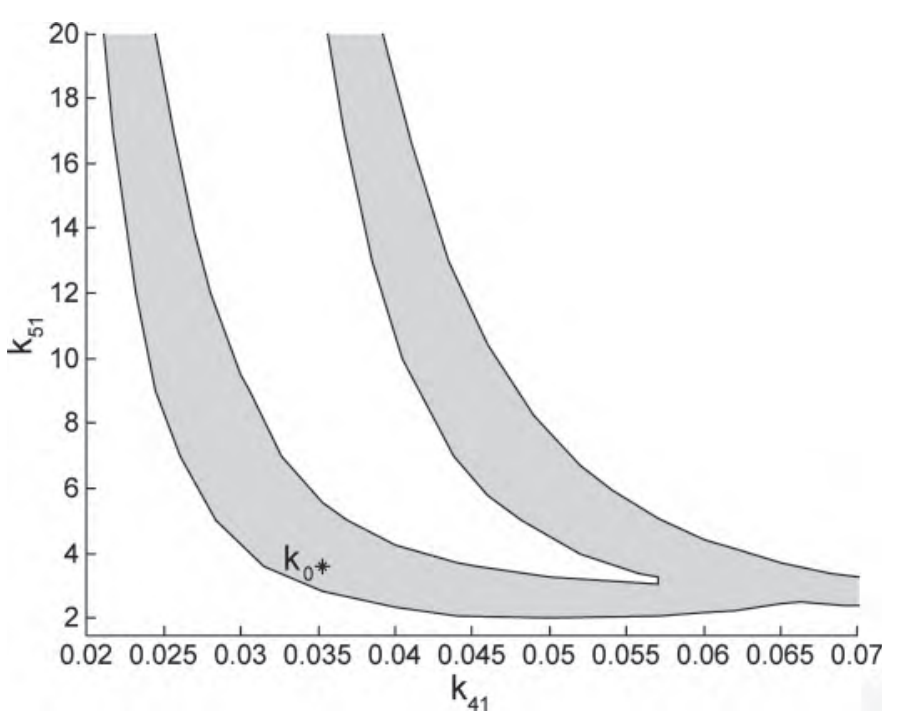

Figure 4. The cross-section of robustness region of the model in the 2-dimensional $\left(k_{41}, k_{51}\right)$ plane (gray area) for parameters 41 and 51 . The nominal parameter set $k_{0}$ is marked by $(*)$. A normal cycle is obtained when $\left(k_{41}, k_{51}\right)$ take values in the gray area, keeping the other parameters fixed at nominal values. Note that the scale for $k_{41}$ and $k_{51}$ is not the same. 
directions 1, 37, 41, and 53 are shown in Figure 5. As can be seen from this figure, in direction 1, components $41,45,51$, and 52 have a relatively high value. Thus, in direction 1, parameters 41 (maximum increase of CL stimulated by itself), 45 (proportionality factor of CL in P4 increase), 51 (maximum increase of OTR stimulated by $\mathrm{P} 4$ ), and 52 (threshold of $\mathrm{P} 4$ to stimulate OTR increase) are more perturbed than the others. In direction 41, components 1 and 41 are dominant; thus, parameter 1 (synthesis rate constant of $\mathrm{GnRH}$ in the hypothalamus) and 41 are perturbed most. In all other perturbation directions, only 1 component is dominating. For each perturbation direction, the maximal variations are given, both in positive and negative directions, for which the system is still robust (i.e., for which the model predicts a normal cycle; Figure 6). For the 120 perturbation directions (up or down for 60 parameters), it occurred 9 times that the perturbation was stopped because one of the parameters passed zero, 6 times that P4 crossed the upper boundary, 10 times that delayed luteolysis was detected, 45 times that delayed ovulation was detected, and 50 times that delayed luteolysis as well as delayed ovulation occurred in a series of successive cycles (Figure 6). The minimum duration $(9 \mathrm{~d})$ of the luteal phase was never a reason to stop the perturbation. The plots of E2 and LH were always in line with the plot of $\mathrm{P} 4$ at the boundary of the robustness region. From Figure 6, it can be noticed that the robustness region is narrowest along the directions 1 and 41. In Figure 5, it is shown that direction 1 is dominated by parameters $41,45,51$, and 52 , so the system is very sensitive to simultaneous changes in these parameters. A similar conclusion holds for direction 41, which is dominated by parameters 1 and 41. Thus, 2 scenarios were found where the system becomes nonrobust immediately: for the first scenario (in direction 1), parameters $41,45,51$, and 52 should be dominating in the perturbation, and for the second scenario (in direction 41), parameters 1 and 41 should be dominating in the perturbation (Table 1). In all other perturbation directions, only 1 parameter is dominating in the perturbation, but it is important to realize that these directions cannot be considered as single parameter changes because the transition in qualitative behavior of the model is also due to the slight changes in other parameter values. Summarizing, the result in Figure 6 shows some specific configurations in multiparameter perturbation that lead the system to a nonrobust behavior immediately. In particular, we found that the system is very sensitive to parameter 41 and changes in this parameter must be very limited to preserve normal behavior of the system.

\section{Progesterone Patterns Associated with Delayed Ovulation and Delayed Luteolysis}

For 105 out of the 120 perturbation directions, the system became nonrobust because of the occurrence of delayed luteolysis or delayed ovulation, or both (Figure 6). When the luteal phase was longer than $19 \mathrm{~d}$ (i.e., when delayed luteolysis was detected), cycles contained 4 waves of follicle growth (indicated by E2 levels; Figure 7). The luteal phase length increased further when the perturbation was continued, resulting in an increasing number of waves per cycle. When the interluteal interval was longer than $12 \mathrm{~d}$, and delayed ovulation was detected, the LH surge was delayed (Figure 8). A pattern of successive cycles with delayed ovulation was never observed. Although parameter configurations resulting in delayed luteolysis could show normal oscillatory behavior, though with more than 3 waves of follicle development per cycle, the observed parameter configurations resulting in delayed ovulation always had irregular P4 patterns (as in Figure 8).

For 36 out of the 60 directions, it was observed that when perturbation in a negative direction was stopped because of delayed ovulation, perturbation in a positive direction stopped because within a series of successive cycles, some cycles with delayed ovulation and some with delayed luteolysis occurred (Figure 6). A typical series of cycles with both delayed ovulation and delayed luteolysis is shown in Figure 8, where the first cycle is normal, the next shows delayed luteolysis, and the third shows delayed ovulation. It also happened that one or more normal cycles occurred in between a cycle with delayed luteolysis and a cycle with delayed ovulation. Interestingly, for these 36 directions, continued perturbation in negative direction resulted again in a series of estrous cycles with normal oscillatory behavior, though with 2 waves of follicular development instead of 3 , whereas continued perturbation in a positive direction resulted in a series of cycles with delayed luteolysis, containing 4 follicular waves (as in Figure 7). Summarizing, for these 36 directions a shift from a cycle with 2 follicular waves to a cycle with 3 follicular waves (i.e., the nominal settings) involves a transition period with irregular $\mathrm{P} 4$ patterns; likewise for a shift from 3 to 4 waves (i.e., delayed ovulation) per cycle (Table 2). As observed already in the cross-section of the robustness region in the 2-dimensional $\left(k_{41}, k_{51}\right)$ plane (Figure 4$)$, configurations with irregular hormone patterns could, thus, lie in between configurations with normal oscillatory behavior. 

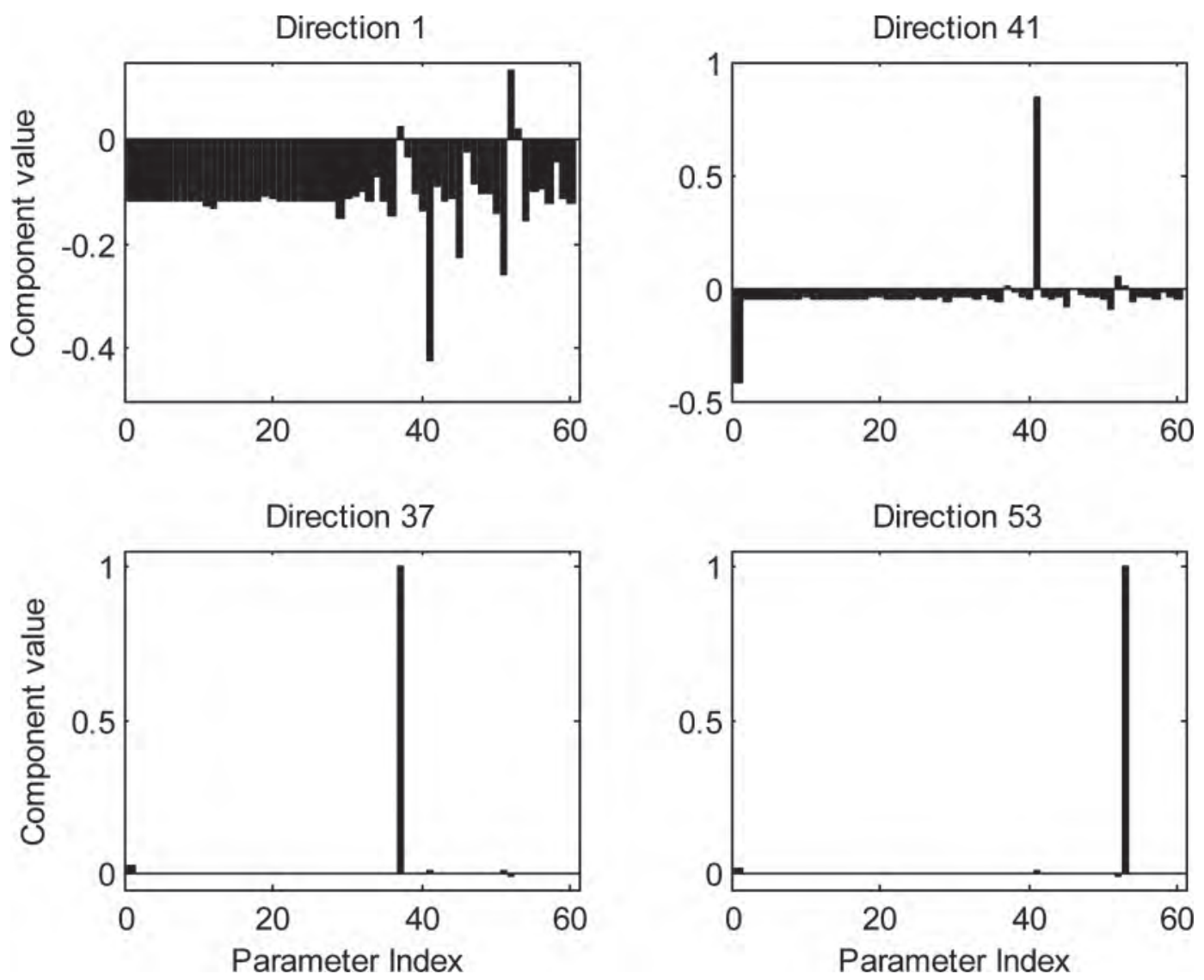

Figure 5. Components of 4 perturbation vectors (selected from the in total 60 perturbation vectors). In perturbation direction 1 , components $41,51,45$, and 52 are considerably larger than the others. In direction 41, components 1 and 41 are clearly dominating. In all other directions only 1 component is dominating, as shown for directions 37 and 53. Note the scale differences between the panels.

\section{DISCUSSION}

\section{Method of Robustness Region Estimation}

In the present paper, the robustness region in parameter space of a mathematical model of the bovine estrous cycle was estimated. Within the robustness region a normal estrous cycle is predicted, and on the boundaries the dynamic behavior of the cycle undergoes a transition, indicating that the system is no longer robust when the parameters are perturbed further. A first approximation for the robustness region in the 60-dimensional parameter space was found by perturbing the nominal parameters set along 60 perpendicular directions. Because each perturbation direction can be followed in positive and negative direction, in this way 120 points on the boundary of the robustness region were obtained. From the checked output curves for E2 and LH, it appeared that evaluation of the system based on $\mathrm{P} 4$ profiles only is a reliable method to detect for which parameter region a normal estrous cycle is simulated, because these curves were in accordance with the P4 profile. Parameter perturbation resulted in delayed ovulation or delayed luteolysis for as many as 105 out of the 120 points on the boundary of the robustness region. In dairy cows, delayed ovulation and delayed luteolysis are associated with the formation of cystic follicles and persistent CL, respectively (Darwash et al., 1998), which is indeed a common cause of reproductive failure. The fact that other cycle abnormalities were not observed might reflect the chosen criteria for a normal cycle used in this study.

\section{Course of Progesterone Patterns Along Perturbation Directions}

In line with experimental data described in the literature, simulated cycles with delayed ovulation or delayed luteolysis continued to have follicular waves (Amer and Mahdi, 2008). When the model predicted a pattern of successive cycles with delayed luteolysis, 


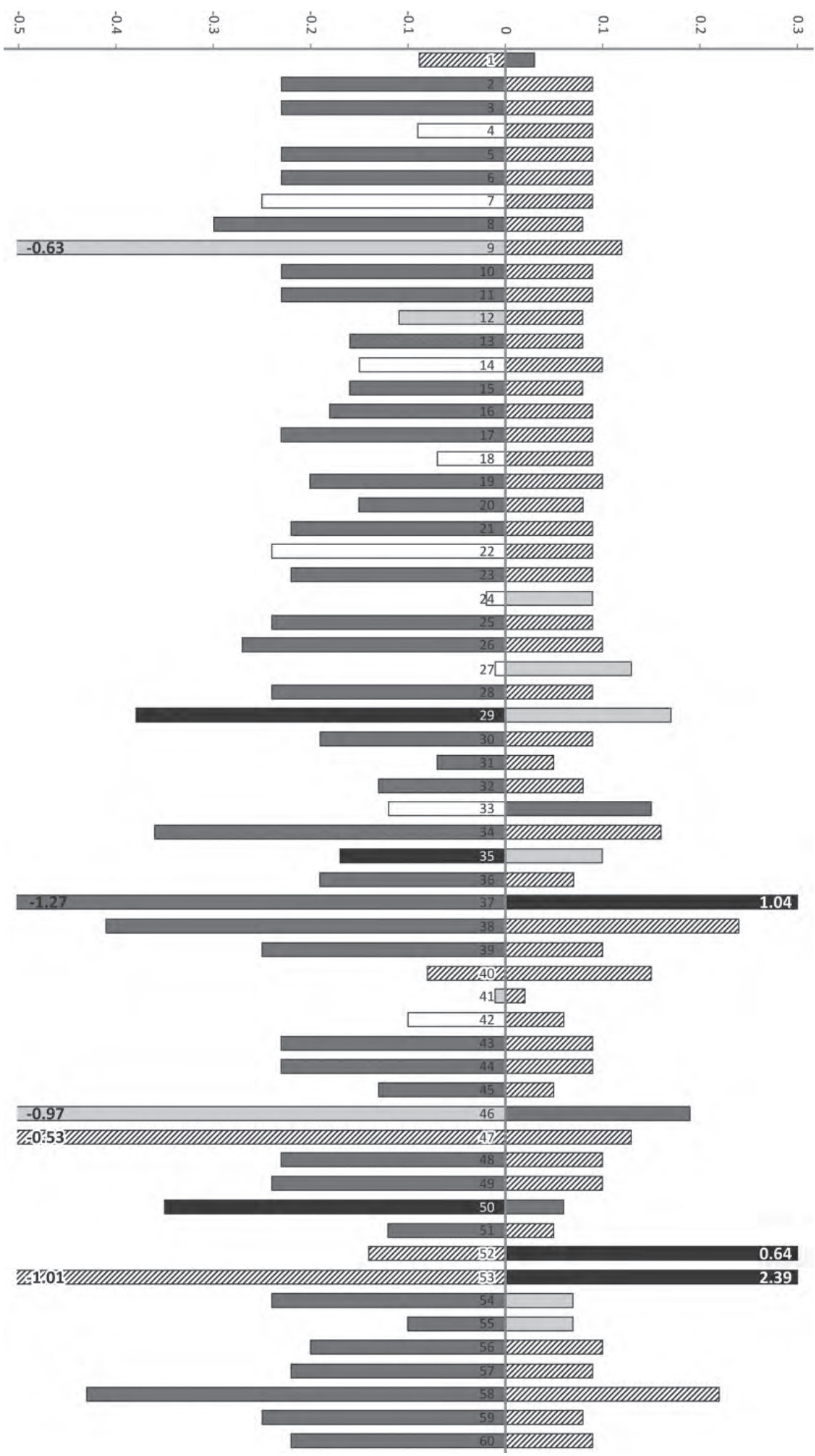

Figure 6. For each of the 60 perturbation directions it was checked how far the direction could be followed before the boundary of the robustness region is reached. All directions are followed in both positive and negative direction, starting at the nominal set of parameter values. The lengths of the bars indicate how far a perturbation direction can be followed in negative and positive directions such that a normal cycle is found. Perturbation was stopped when one of the parameters passed zero (white bars), when P4 levels are above 2.00 (black bars), when delayed ovulation (dark gray) or delayed luteolysis (light gray) was detected, or when delayed ovulation as well as delayed luteolysis was detected in a series of successive cycles (striped). 
Table 1. Five parameters for which the (combined) change in values immediately leads to an irregular cycle

\begin{tabular}{llc}
\hline Parameter $^{1}$ & Description $^{2}$ & $\begin{array}{c}\text { Perturbed in direction } 1 \\
\text { or direction } 41\end{array}$ \\
\hline Par 1 & Synthesis rate constant of GnRH in the hypothalamus & 1 \\
Par 41 & Maximum increase of CL stimulated by itself & 1,41 \\
Par 45 & Proportionality factor of CL in P4 increase & 41 \\
Par 51 & Maximum increase of OTR stimulated by P4 & 41 \\
Par 52 & Threshold of P4 to stimulate OTR increase & 41 \\
\hline
\end{tabular}

${ }^{1} \mathrm{Par}=$ parameter.

${ }^{2} \mathrm{CL}=$ corpus luteum function; $\mathrm{P} 4=$ progesterone; OTR $=$ oxytocin receptor.

the estrous cycle showed regular oscillatory behavior but contained 4 waves of follicular development due to a prolonged luteal phase. Probably this should not be considered as a pathological condition, because estrous cycles with 4 follicular waves are occasionally observed in healthy fertile cows. However, it is clear that normal cyclicity is impaired when within a series of successive cycles some cycles with delayed ovulation and some with delayed luteolysis occurred. The course of $\mathrm{P} 4$ profiles described in Table 2 shows that parameter configurations of delayed ovulation and delayed luteolysis can lie in between configurations with normal oscillatory behavior for different numbers of follicular waves. Although it is difficult to define if this is a likely physiological configuration in cows, this could represent a situation of resumption of normal cyclicity (e.g., after parturition). The observed irregular patterns in the model where delayed ovulation as well as delayed luteolysis occur suggest that delayed ovulation and delayed luteolysis in reality could have the same cause and are characterized by irregular hormone patterns. These irregular hormone patterns might explain why it is difficult in practice to give a clear pathology of cystic ovaries and why outcomes of treatments are variable (Garverick, 1997).

\section{Factors That May Lead to Delayed Ovulation and Delayed Luteolysis}

Perturbation vectors responsible for significant qualitative transitions in the behavior of the system, causing nonrobustness, mirror the combined effects of several perturbed parameters. However, perturbation along 2 specific vectors was found to cause nonrobustness of the system immediately: for directions 1 and 41, a very small perturbation in a negative direction resulted in delayed luteolysis (and for direction 1 in delayed ovulation as well), and a very small perturbation in a positive direction resulted in delayed ovulation. In direction 1 , parameters 41 (maximum increase of CL stimulated by itself), 45 (proportionality factor of CL in P4 increase), 51 (maximum increase of OTR stimulated by P4), and 52 (threshold of $\mathrm{P} 4$ to stimulate OTR increase) are more perturbed than the others. In direction 41, parameters 1 (synthesis rate constant of GnRH in the hypothalamus) and 41 are most perturbed. This suggests that these parameters strongly determine the occurrence of P4 patterns that are associated with delayed ovulation and delayed luteolysis in the model.

Synthesis rate constant of $\mathrm{GnRH}$ in the hypothalamus (parameter 1) affects the amount of hypothalamic

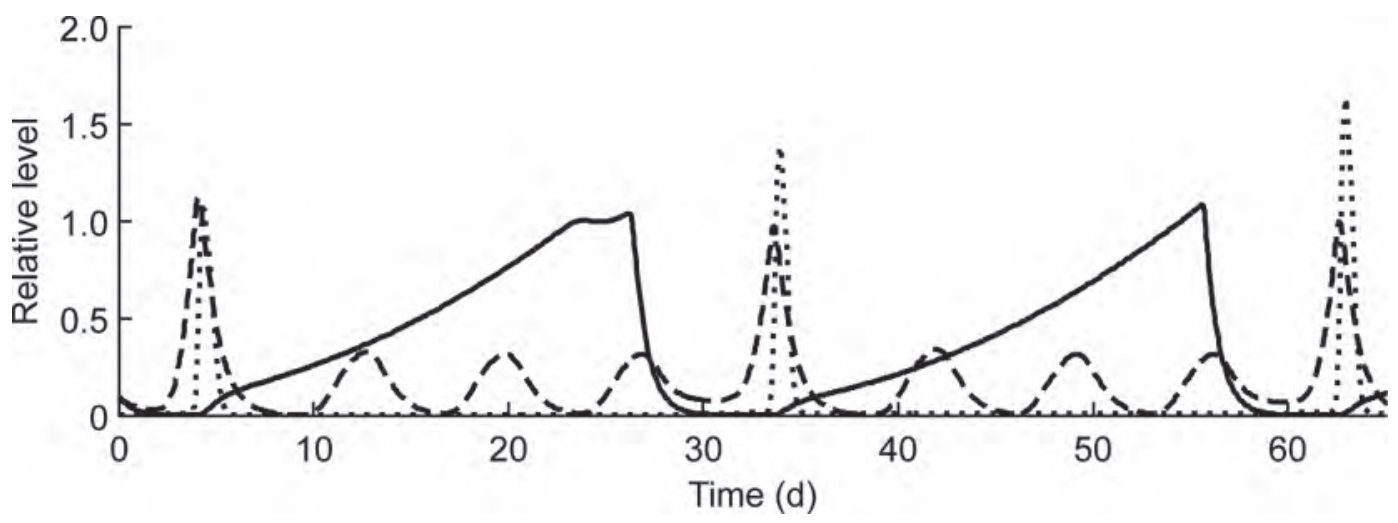

Figure 7. Relative levels of progesterone (P4; solid line), estradiol (E2; dashed line), and LH (dotted line) at perturbation direction $k_{0}+$ $0.12 \times v_{9}$, where $k_{0}=$ nominal parameter set and $v_{9}=$ perturbation direction 9 , representing the main pattern of model output curves associated with delayed luteolysis. 


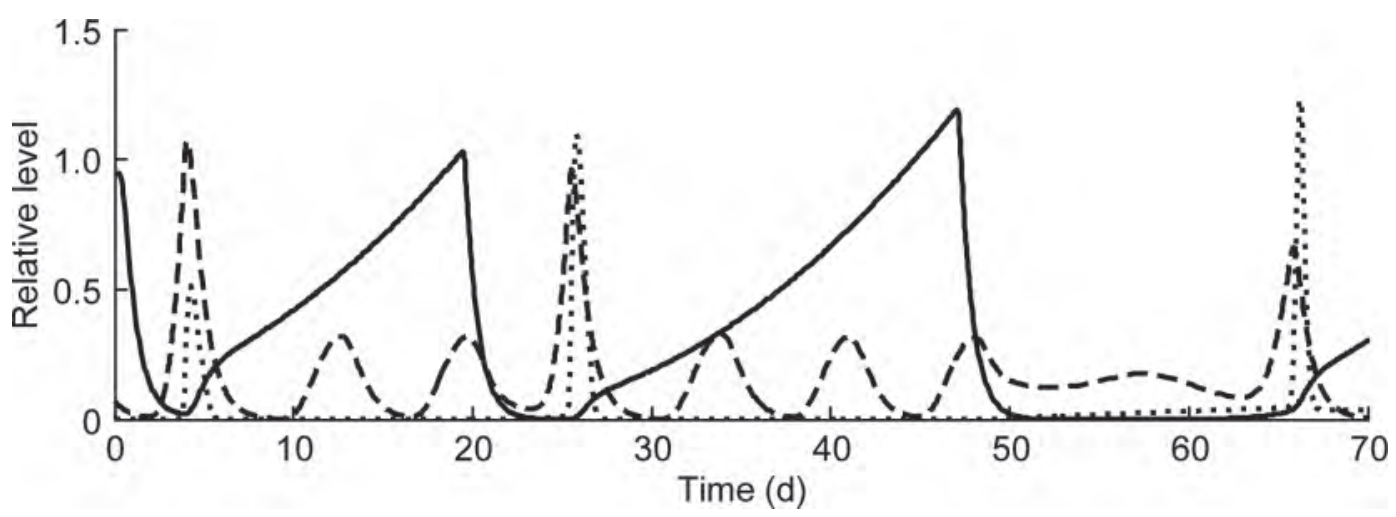

Figure 8. Relative levels of progesterone (P4; solid line), estradiol (E2; dashed line), and LH (dotted line) at perturbation direction $k_{0}+0.10$ $\times v_{39}$, where $k_{0}=$ nominal parameter set and $v_{39}=$ perturbation direction 39,representing the main pattern of model output curves associated with delayed ovulation.

GnRH that is produced. A perturbation of $\mathrm{GnRH}$ synthesis could inhibit the surge of GnRH and subsequently of LH, thereby blocking ovulation and inducing the formation of a cystic follicle. Delayed ovulation, which indicates cystic follicle formation, occurred in the model in absence of an LH surge. This phenomenon has also been observed in practice and may be the result of hypothalamic insensitivity to E2 caused by inadequate exposure to P4 (Gümen and Wiltbank, 2002). Hypothalamic content of $\mathrm{GnRH}$ is lower in cows with cysts (Garverick, 1997). Ovulation of a cystic follicle after GnRH treatment usually does not occur (Garverick, 1997), but in the model we could not check whether the GnRH/LH surge following delayed ovulation causes ovulation or only luteinization of the follicle, because the event of ovulation is not separately included in the model.

Maximum increase of CL stimulated by CL itself (parameter 41) in the model captures physiological processes such as local effects of OT, $\mathrm{PGF}_{2 \alpha}$, and noradrenaline on CL development and maintenance (Skarzynski et al., 2008). From a biological perspective, this could translate to the fact that a proper functioning of the CL (i.e., sufficient P4 production) is crucial for normal cyclicity. Maximum increase of OTR stimulated by $\mathrm{P} 4$ (parameter 51) and threshold of $\mathrm{P} 4$ to stimulate
OTR increase (parameter 52) affect the time point of luteolysis. It may seem obvious and not surprising that parameters regulating the time point of luteolysis are involved in the occurrence of $\mathrm{P} 4$ profiles associated with delayed ovulation and delayed luteolysis. However, it is likely that in many other perturbation directions the change in $\mathrm{P} 4$ profiles is the indirect effect of many small changes in other parameters. Moreover, all model components are connected and more or less affecting each other, which makes it hard to predict beforehand which specific mechanisms are involved in the development of cystic ovaries. Experiments to verify model predictions would require measurements during successive estrous cycles because it is unpredictable when development of cystic ovaries occurs. Further, such experiments should go beyond blood sampling because this would only identify the occurrence and not the underlying causes of cystic ovaries, which according to the simulation results are more likely at a cellular level.

\section{CONCLUSIONS}

The method of robustness region estimation used in the present study is an effective tool to find parameter configurations that lead to specific endocrine profiles in a model of the bovine estrous cycle, such as P4 profiles

Table 2. Types of progesterone (P4) patterns observed for perturbation of 36 out of the 60 directions

\begin{tabular}{ll}
\hline Parameter configuration & P4 pattern and follicular wave pattern \\
\hline Continued perturbation in negative direction & Normal cycles without variation ${ }^{1}$ with 2 follicular waves \\
Perturbation in negative direction & Variable cycles with delayed ovulation \\
Nominal set & Normal cycles without variation with 3 follicular waves \\
Perturbation in positive direction & Variable cycles with delayed ovulation and delayed luteolysis ${ }^{2}$ \\
Continued perturbation in positive direction & Successive cycles of delayed luteolysis without variation \\
\hline
\end{tabular}

${ }^{1}$ Progesterone patterns are similar in each successive cycle.

${ }^{2}$ Within a series, some cycles have delayed ovulation and some have delayed luteolysis. 
associated with delayed ovulation and delayed luteolysis. Thereby, the model can help to generate hypotheses regarding the mechanisms and predisposing factors involved in the development of cystic ovaries. The simulation results presented here support that mechanisms regulating CL functioning, luteolytic signals, and $\mathrm{GnRH}$ synthesis are likely to be involved in the development of cystic ovaries. In the multidimensional parameter space, areas exist in which the parameter configurations resulted in normal cycles. These areas may be separated by areas in which irregular cycle patterns occurred (see Figure 4 for an example). These irregular patterns thus mark the transition from one stable (normal) situation to another. Interestingly, within a series, some cycles had delayed ovulation and some had delayed luteolysis in these patterns. The onset of cyst development in cows is currently unpredictable, but the model predictions presented in this study indicate some candidate mechanisms to consider for further investigation.

\section{ACKNOWLEDGMENTS}

These results were obtained through IP/OP: Systems Biology (Wageningen, the Netherlands), financially supported by the Dutch Ministry of Agriculture, Nature and Food Quality (The Hague, the Netherlands; BAS no. 4434660700). C. Stötzel was supported by the DFG Research Center Matheon "Mathematics for Key Technologies" in Berlin, Germany.

\section{REFERENCES}

Amer, H. A., and A. B. Mahdi. 2008. Hormonal profiles associated with treatment of cystic ovaries with $\mathrm{GnRH}$ and $\mathrm{PGF}_{2 \alpha}$ with and without CIDR in dairy cows. Livest. Res. Rural Develop. 20:52.
Apri, M., J. Molenaar, M. de Gee, and G. van Voorn. 2010. Efficient estimation of the robustness region of biological models with oscillatory behavior. PLoS ONE 5:e9865.

Boer, H. M. T., S. Röblitz, C. Stötzel, R. F. Veerkamp, B. Kemp, and H. Woelders. 2011a. Mechanisms regulating follicle wave patterns in the bovine estrous cycle investigated with a mathematical model. J. Dairy Sci. 94:5987-6000.

Boer, H. M. T., C. Stötzel, S. Röblitz, P. Deuflhard, R. F. Veerkamp, and $\mathrm{H}$. Woelders. 2011b. A simple mathematical model of the bovine estrous cycle: Follicle development and endocrine interactions. J. Theor. Biol. 278:20-31.

Braw-Tal, R., S. Pen, and Z. Roth. 2009. Ovarian cysts in high-yielding dairy cows. Theriogenology 72:690-698.

Darwash, A. O., G. E. Lamming, and J. A. Woolliams. 1998. Identifying heritable endocrine parameters associated with fertility in postpartum dairy cows. Pages 40-54 in Proc. International Workshop on Genetic Improvement of Functional Traits in Cattle; Fertility and Reproduction. Vol. 18. Interbull, Grub, Germany.

Garmo, R. T., A. D. Martin, E. Thuen, Ø. Havrevoll, H. Steinshamn, E. Prestløkken, A. Randby, M. Eknæs, A. Waldmann, and O. Reksen. 2009. Characterization of progesterone profiles in fall-calving Norwegian Red cows. J. Dairy Sci. 92:4919-4928.

Garverick, H. A. 1997. Ovarian follicular cysts in dairy cows. J. Dairy Sci. 80:995-1004

Ghaemi, R., J. Sun, P. A. Iglesias, and D. Del Vecchio. 2009. A method for determining the robustness of bio-molecular oscillator models. BMC Syst. Biol. 3:95.

Gümen, A., and M. C. Wiltbank. 2002. An alteration in the hypothalamic action of estradiol due to lack of progesterone exposure can cause follicular cysts in cattle. Biol. Reprod. 66:1689-1695.

Petersson, K. J., H. Gustafsson, E. Strandberg, and B. Berglund. 2006. Atypical progesterone profiles and fertility in Swedish dairy cows. J. Dairy Sci. 89:2529-2538.

Skarzynski, D. J., G. Ferreira-Dias, and K. Okuda. 2008. Regulation of luteal function and corpus luteum regression in cows: Hormonal control, immune mechanisms and intercellular communication. Reprod. Domest. Anim. 43(Suppl. 2):57-65.

Stötzel, C., J. Plöntzke, and S. Röblitz. 2012. Advances in modelling of the bovine estrous cycle: Administration of PGF2 $\alpha$. Theriogenology http://dx.doi.org/10.1016/j.theriogenology.2012.04.017.

Vanholder, T. 2005. Cystic ovarian follicles in the high yielding dairy cow post partum: Role of metabolic and hormonal adaptations in the pathogenesis. PhD Thesis. Ghent University, Ghent, Belgium.

Vanholder, T., G. Opsomer, and A. de Kruif. 2006. Aetiology and pathogenesis of cystic ovarian follicles in dairy cattle: A review. Reprod. Nutr. Dev. 46:105-119. 
APPENDIX

Table A1. List of model equations ${ }^{1}$

\begin{tabular}{|c|c|}
\hline Equation number & Equation \\
\hline 1 & $\frac{d}{d t} G n R H_{H y p o}(t)=S y n_{G n R H}(t)-\operatorname{Rel}_{G n R H}(t)$ \\
\hline $1 \mathrm{a}$ & $\operatorname{Syn}_{G n R H}(t)=c_{G n R H, 1} \cdot\left(1-\frac{G n R H_{H y p o}(t)}{G n R H_{\text {Hypo }}^{\max }}\right)$ \\
\hline $1 b$ & $\operatorname{Rel}_{G n R H}(t)=\left[H_{1}^{-}(P 4 \& E 2)+H_{2}^{-}(P 4)\right] \cdot G n R H_{H y p o}(t)$ \\
\hline 2 & $\frac{d}{d t} G n R H_{P i t}(t)=\operatorname{Rel}_{G n R H}(t) \cdot H_{3}^{+}(E 2)-c_{G n R H, 2} \cdot G n R H_{P i t}(t)$ \\
\hline 3 & $\frac{d}{d t} F S H_{P i t}(t)=\operatorname{Syn}_{F S H}(t)-\operatorname{Rel}_{F S H}(t)$ \\
\hline $3 \mathrm{a}$ & $\operatorname{Syn}_{F S H}(t)=H_{4}^{-}(\operatorname{Inh})$ \\
\hline $3 \mathrm{~b}$ & $\operatorname{Rel}_{F S H}(t)=\left[b_{F S H}+H_{5}^{+}(P 4)+H_{6}^{-}(E 2)+H_{7}^{+}\left(G n R H_{P i t}\right)\right] \cdot F S H_{P i t}(t)$ \\
\hline 4 & $\frac{d}{d t} F S H_{B l o o d}(t)=\operatorname{Rel}_{F S H}(t)-c_{F S H} \cdot F S H_{B l o o d}(t)$ \\
\hline 5 & $\frac{d}{d t} L H_{P i t}(t)=\operatorname{Syn}_{L H}(t)-\operatorname{Rel}_{L H}(t)$ \\
\hline $5 \mathrm{a}$ & $\operatorname{Syn}_{L H}(t)=H_{8}^{+}(E 2)+H_{9}^{-}(P 4)$ \\
\hline $5 \mathrm{~b}$ & $\operatorname{Rel}_{L H}(t)=\left[b_{L H}+H_{10}^{+}\left(G n R H_{P i t}\right)\right] \cdot L H_{P i t}(t)$ \\
\hline 6 & $\frac{d}{d t} L H_{\text {Blood }}(t)=\operatorname{Rel}_{L H}(t)-c_{L H} \cdot L H_{\text {Blood }}(t)$ \\
\hline 7 & $\frac{d}{d t} \operatorname{Foll}(t)=H_{11}^{+}\left(F_{S H} H_{\text {Blood }}\right)-\left[H_{12}^{+}(P 4)+H_{13}^{+}\left(L H_{\text {Blood }}\right)\right] \cdot$ Foll $(t)$ \\
\hline 8 & $\frac{d}{d t} C L(t)=S F \cdot H_{13}^{+}\left(L H_{\text {Blood }}\right) \cdot$ Foll $(t)+H_{14}^{+}(I O F)-H_{15}^{+}(I O F) \cdot C L(t)$ \\
\hline 9 & $\frac{d}{d t} P 4(t)=c_{C L}^{P 4} \cdot C L(t)^{2}-c_{P 4} \cdot P 4(t)$ \\
\hline 10 & $\frac{d}{d t} E 2(t)=c_{F o l l}^{E 2} \cdot$ Foll $(t)^{2}-c_{E 2} \cdot E 2(t)$ \\
\hline 11 & $\frac{d}{d t} \operatorname{Inh}(t)=c_{\text {Foll }}^{\text {Inh }} \cdot \operatorname{Foll}(t)^{2}-c_{\text {Inh }} \cdot \operatorname{Inh}(t)$ \\
\hline 12 & $\frac{d}{d t} O T R(t)=H_{16}^{+}(P 4)-c_{\text {OTR }} \cdot O T R(t)$ \\
\hline 13 & $\frac{d}{d t} O T(t)=H_{17}^{+}(E 2) \cdot C L(t)^{2}-C_{O T} \cdot O T(t)$ \\
\hline 14 & $\frac{d}{d t} \operatorname{IOF}(t)=H_{18}^{+}\left(P G F_{2 \alpha} \& C L\right)-c_{I O F} \cdot \operatorname{IOF}(t)$ \\
\hline 15 & $\frac{d}{d t} P G F_{2 \alpha}(t)=H_{19}^{+}(O T R \& O T)-c_{P G F_{2 \alpha}} \cdot P G F_{2 \alpha}(t)$ \\
\hline
\end{tabular}

${ }^{1}$ For abbreviation of the notation of Hill functions, we use $H$ (substrate) instead of $m \cdot h[$ substrate $(t) ; T, n]$, where $H^{+}=$positive Hill function, $H=$ negative Hill function, $m=$ parameter that controls the height of the switch of the Hill functions, $t=$ time, $T=$ threshold for change of behavior of the Hill functions, and $n$ is the steepness coefficient. Syn = synthesis; Rel = release; Pit = pituitary; Hypo = hypothalamus; P4 = progesterone; E2 $=$ estradiol; $I n h=$ inhibin $O T=$ oxytocin; $O T R=O T$ receptor; $C L=$ corpus luteum; Foll $=$ follicle; $I O F=$ interovarian factors; $\max =$ maximum; $c=$ rate constant. 
Table A2. List of Hill functions ${ }^{1}$

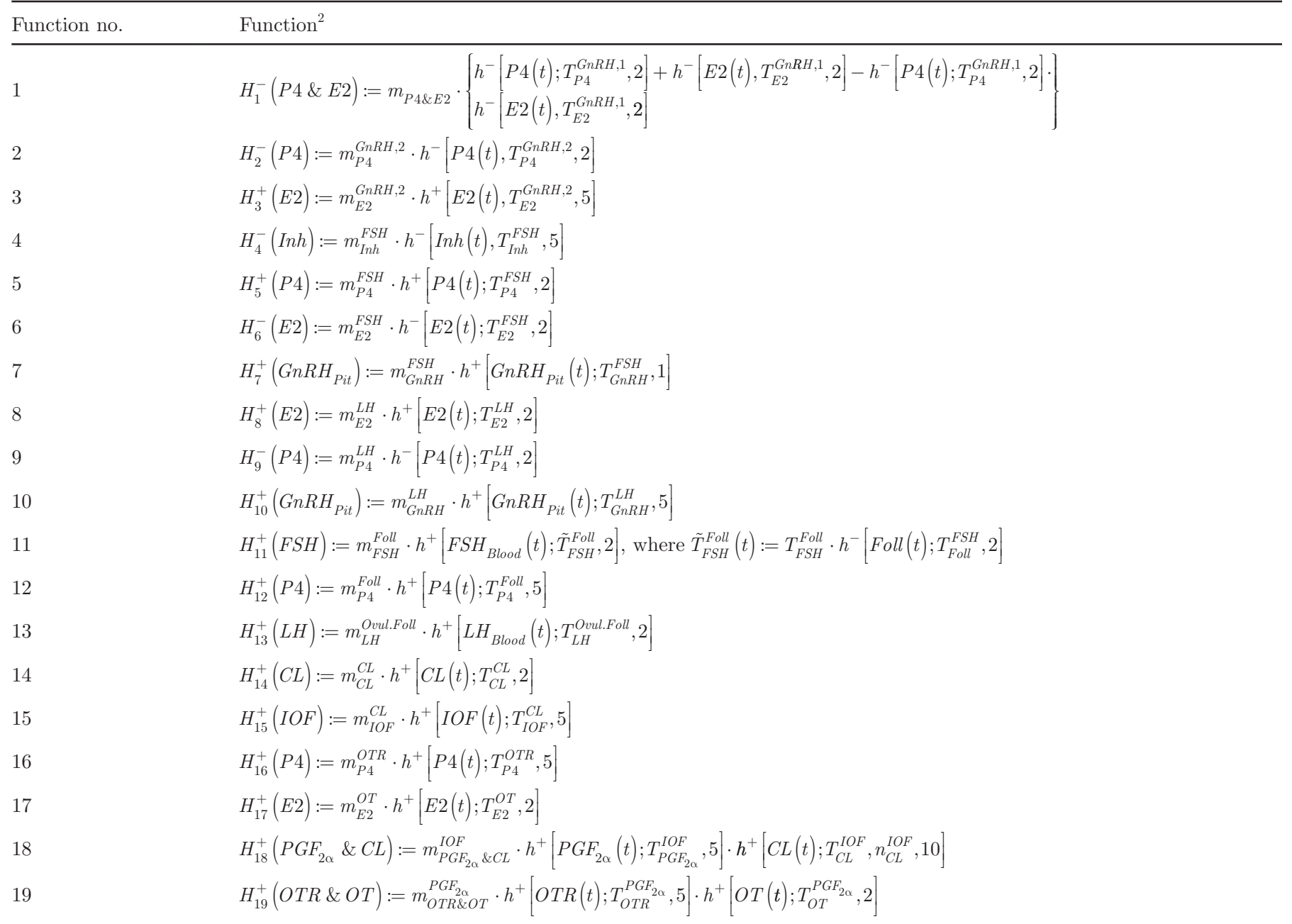

${ }^{1}$ In the differential equations, Hill functions were used for the modeling of inhibitory and stimulatory effects of hormones. A Hill function is a sigmoidal function between 0 and 1 , which switches at a specified threshold from one level to the other with a specified steepness

${ }^{2}$ The notation of Hill functions is $m \cdot h[\operatorname{substrate}(t) ; T, n]$, where $H^{+}=$positive Hill function, $H=$ negative Hill function, $m=$ parameter that controls the height of the switch of the Hill function, $t=$ time, $T=$ threshold for change of behavior of the Hill functions, and $n$ is the steepness coefficient. $\mathrm{E} 2=$ estradiol; P4 = progesterone; Inh $=$ inhibin; OT $=$ oxytocin; OTR $=$ OT receptor; IOF $=$ interovarian factors; CL $=$ corpus luteum; Foll $=$ follicle. 
Table A3. List of parameters and parameter values of the initial 3-wave cycle

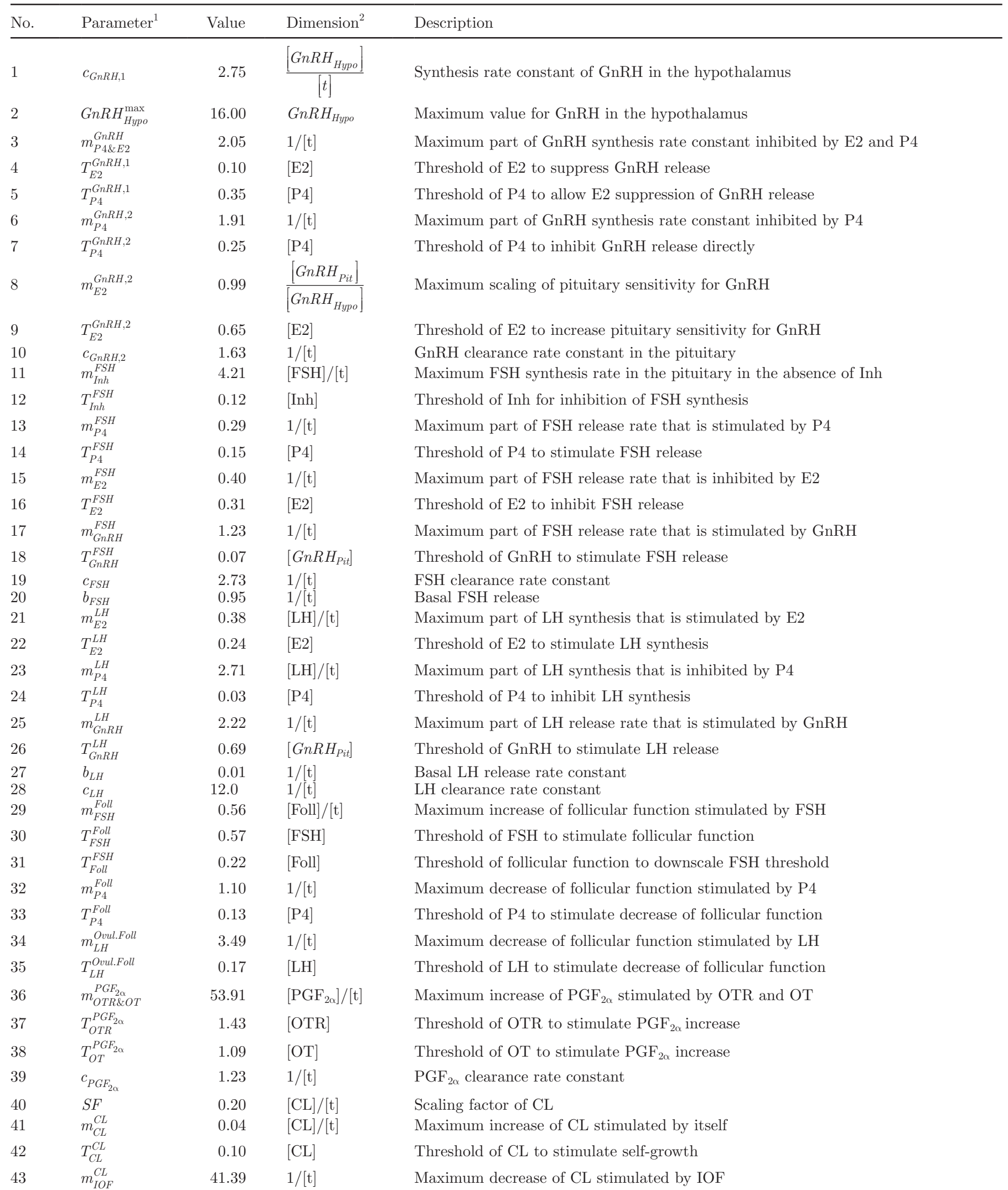


Table A3 (Continued). List of parameters and parameter values of the initial 3-wave cycle

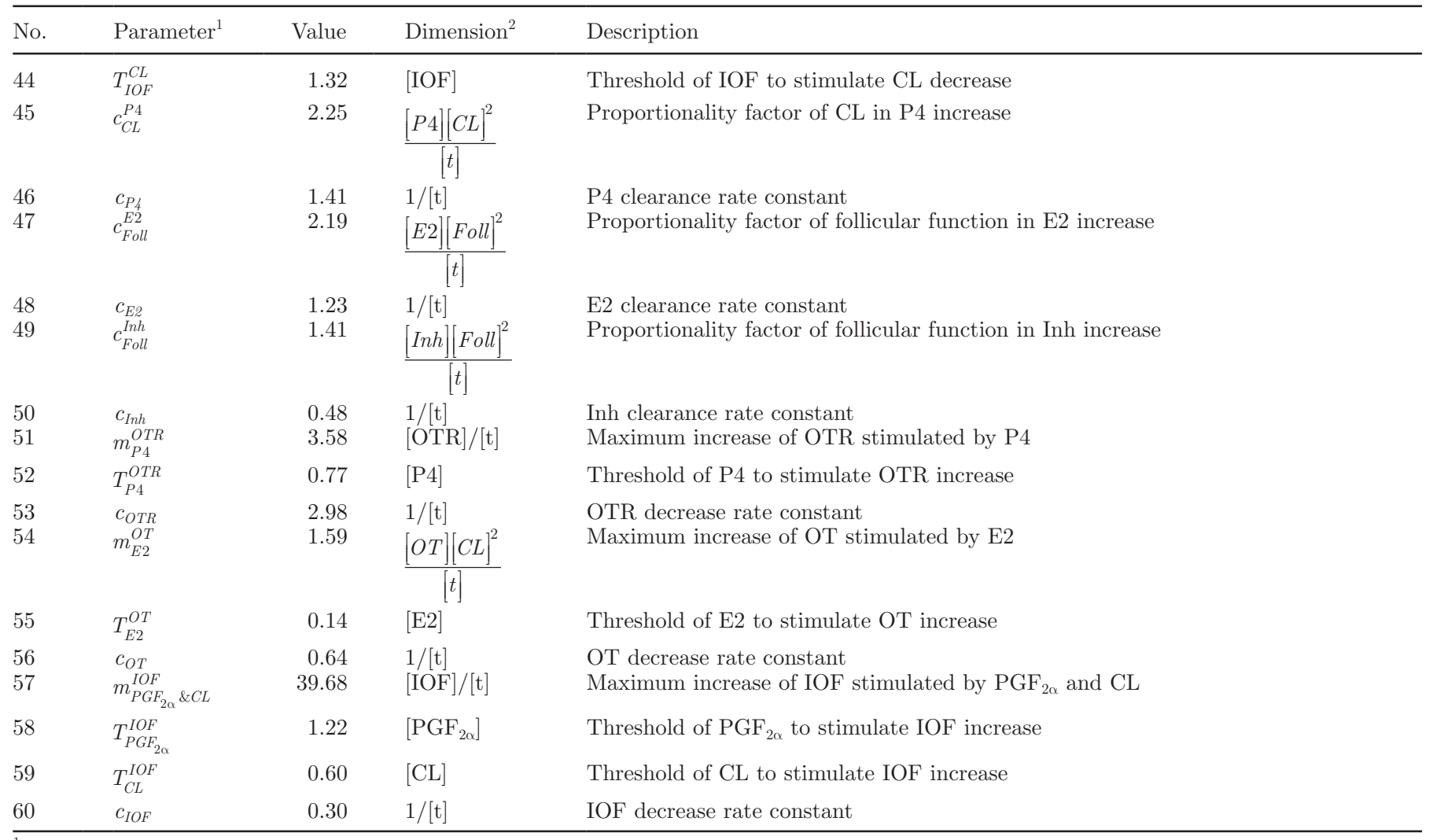

${ }^{1} \max =$ maximum; $m=$ parameter that controls the height of the switch of the Hill function; $\mathrm{P} 4=$ progesterone; E2 = estradiol; $T=$ threshold for change of behavior of the Hill functions; $b=$ basal rate constant; $c=$ clearance rate constant; Foll = follicle; Ovul.Foll $=$ ovulatory follicle; $C L=$ corpus luteum; $I O F=$ interovarian factors; $I n h=$ inhibin; $O T=$ oxytocin; $O T R=O T$ receptor.

${ }^{2}$ Hypo $=$ hypothalamus; $t=$ time; Pit $=$ pituitary. 